\title{
The direct and indirect effects of corruption on motor vehicle crash deaths.
}

\begin{abstract}
Recent empirical research has found that there is an inverted U-shaped or Kuznets relationship between income and motor vehicle crash (MVC) deaths, such that MVC deaths increase as national income increases and decrease after reaching a critical level. Corruption has been identified as one of the underlying factors that could affect this relationship, primarily by undermining institutional development and effective enforcement schemes. The total effect of corruption can be decomposed into two components, a direct and an indirect effect. The direct effect measures the immediate impact of corruption on MVC deaths by undermining effective enforcement and regulations, while the indirect effect captures the impact of corruption on hindering increases in per capita income and the consequent impact of reduced income on MVC deaths. By influencing economic growth, corruption can lead to an increase or decrease in MVC deaths depending on the income level. Using data from 60 countries between 1982 and 2003, these effects are estimated using linear panel and fixed effects negative binomial models. The estimation results suggest that corruption has different direct effects for less developed and highly developed countries. It has a negative (decreasing) effect on MVC deaths for less developed countries and a positive (increasing) effect on MVC deaths for highly developed countries. For highly developed countries, the total effect is positive at lower per capita income levels, but decreases with per capita income and becomes negative at per capita income levels of about US\$ 38,248. For less developed countries, the total effect is negative within the sample range and decreases with increased per capita income. In summary, the results of this study suggest that reduction of corruption is likely a necessary condition to effectively tackle road safety problems.
\end{abstract}

Keyword: Road fatalities; Corruption; Kuznets Curve; Road safety; Vehicle; Motor vehicle crash. 